\title{
Structural Analysis of Predicted HIV-1 Secis Elements
}

\author{
Paushali Roy ${ }^{*}$, Sayak Ganguli, Pooja Sharma, Protip Basu, Abhijit Datta
}

DBT Centre for Bioinformatics, Presidency University, Kolkata, India.

E-mail: "paushali.06@gmail.com

Received August 30 ${ }^{\text {th }}, 2011$; revised October $6^{\text {th }}, 2011$; accepted October $19^{\text {th }}, 2011$.

\begin{abstract}
Incorporation of Selenocysteine into protein requires an RNA structural motif, SECIS (Selenocysteine insertion sequence) element that, along with other factors, demarcates UGA-Sec from the UGA termination codon, for expression of Selenoproteins (in case of eukaryotes). It has been predicted that during HIV infection, several functional viral selenoproteins are expressed and synthesis of these viral selenoproteins deplete the selenium level of the host. It might be that even the viral genome has the SECIS elements in their Selenoprotein $m R N A$, and during infection, the host cellular machinery is transformed in such a way that the human Sec tRNA binds to the viral Selenoprotein mRNA, instead of binding to its own Selenoprotein mRNA, thus leading to expression of viral selenoproteins. This hypothesis was tested in this study by identifying the SECIS elements in the HIV-1 genome and further predicting their secondary and tertiary structures. We then tried to dock these tertiary structures with human Sec tRNA. Here we report putatively the presence of 3215 SECIS elements in the HIV-1 genome and that the human Sec RNA $^{\text {sec }}$ binds to the viral SECIS elements present in the viral selenoprotein mRNA. Based on an earlier finding, it was observed that atoms of A8 and U9, which present in human Sec tRNA, are the possible key sites for binding.
\end{abstract}

Keywords: Selenocysteine, Selenium, SECIS Element, Selenoprotein, Human Sec tRNA ${ }^{\text {sec }}$, UGA Codon

\section{Introduction}

Selenium, an essential micronutrient, is a natural component of selenium dependent enzymes, and in most of these it occurs in the amino acid selenocysteine, that is present in the catalytic centers of the proteins [1]. These selenium dependent enzymes called selenoproteins include one or more Selenocysteine residues, where selenium acts an antioxidant [2]. Selenium plays an important role in the proper functioning of the immune system and inhibiting the progression of HIV infection to AIDS. It is required for the activity of the enzyme glutathione peroxidase, and deficiency in selenium may cause myopathy, cardiomyopathy and immune dysfunction [3].

Selenoproteins such as glutathione peroxidases, thioredoxin reductases, and iodothyronine deiodinases are involved in redox reactions [4]. At the physiological level, these enzymes are involved in diverse metabolic and physiological functions ranging from antioxidant defense to fertility, muscle development and function, thyroid hormone metabolism, and immune function [5].

Expression of the selenoproteins requires the incorporation and biosynthesis of the amino acid Selenocysteine (reviewed in Atkins \& Gesteland, 2000). Selenocysteine is the $21^{\text {st }}$ amino acid in the genetic code and is encoded by the codon UGA that is generally a termination codon. Certain factors have been found in eukaryotes that mediate the biosynthesis of Selenocysteine and thus the expression of selenoproteins [6,7]. One of the major factor are the SECIS elements, an RNA structural motif, that have been found in the 3' UTR of the eukaryotic selenoprotein mRNA.

The 3' and 5' untranslated regions of the HIV-1 genome have all the RNA motifs concentrated within it, these include internal ribosome entry sites, packaging signals, pseudoknots, transfer RNA mimics, ribosomal frameshift motifs, and cis-regulatory elements $[8,9]$. In the human immunodeficiency virus (HIV), RNA structures activate transcription, initiate reverse transcription, facilitate genomic dimerization, direct HIV packaging, manipulate reading frames, regulate RNA nuclear export, signal polyadenylation, and interact with viral and host proteins [9-13]. Most potential regulatory structures within the HIV-1 genome are uncharacterized raising the possibility of new RNA structure-mediated regulation to be identified [14]. 
It has been reported that during HIV infection the level of selenium in the host, decreases and expression of viral selenoproteins increases. Also, it has been proposed that HIV-1 may encode several selenoproteins one of which has significant sequence similarity to GPx that is a mammalian selenoprotein [15].

Selenocysteine insertion sequence (SECIS) element has not yet been identified in the HIV genome by either biologic or computational methods. Sequence analysis has identified locations in HIV-1 strain HXB2 where SECIS element could exist [16].

The aim of this study is to identify the plausible SECIS elements in the HIV-1 genome and deduce their role in the deficiency of selenium and increased expression of viral selenoproteins during HIV infection.

The results obtained showed that, indeed there are SECIS elements present in the HIV-1 genome and the human Sec $\mathrm{tRNA}^{\mathrm{Sec}}$ binds to the viral selenoprotein mRNA, wherein possibly the key residues are the atoms of A8 and U9 which are involved in stability of the binding.

We hypothesize that during HIV infection when translation occurs, the human Selenocysteine $\operatorname{tRNA}^{\mathrm{Sec}}$ binds to the viral selenoprotein mRNA that has the presence of SECIS elements. Thus Selenocysteine would get incorporated in the growing polypeptide chain, utilizing the host's selenium, and will lead to the expression of viral selenoproteins instead of human selenoproteins.

\section{Methods}

We first retrieved 847 complete genome sequences of the HIV-1 genome from the NCBI database. The RNA regulatory motifs for all these sequences were then obtained using a stacking energy thermodynamic model based on Bayesian statistics for identifying the homologs of Regulatory RNA motifs and elements against an input mRNA sequence. The full process of a typical Bayesian analysis can be roughly described as consisting of three main steps: 1) setting up a full probability model that includes all the variables so as to capture the relation- ship among these variables; 2) summarizing the findings for particular interests by appropriate posterior distributions; 3) evaluating the appropriateness of the model and suggesting improvements [17].

A standard procedure for carrying out step 1) is to first write down the likelihood function, i.e., the probability of the observed data given the unknowns, and multiply it by a prior distribution, i.e., a distribution for all the unobserved variables (typically unknown parameters). The joint probability is represented as joint $=$ likelihood prior, i.e.,

$$
p(y, \theta)=p(y \mid \theta) p(\theta)
$$

where the prior distribution reveals what is known about the parameter without the knowledge of the data. Bayesian inference is drawn by examining the probability of all possible values of the parameter after considering the data. Accordingly, step 2) is completed by obtaining the posterior distribution:

$$
p(\theta \mid y)=\frac{p(\theta \mid y)}{p(y)}=\frac{p(y \mid \theta) p(\theta)}{p(y)} \propto p(y \mid \theta) p(\theta)
$$

where the posterior distribution tells us what is known about y given knowledge of the data.

Both sequence homologs and structural homologs of regulatory RNA motifs could be identified. In this work the basic focus was on the RNA structural motif named SECIS (Selenocysteine Insertion Sequence) element.

Our next in-silico experiment was performed on the same set of HIV-1 genome sequences to specifically identify the SECIS elements, if present, in the genome. This was done using a computational tool based on a SECIS consensus model the key feature of which is a conserved guanosine in a small apical loop of the properly positioned structure [18].

Using the sequences of the SECIS elements obtained, we designed their secondary structures based on the RNA secondary structure (folding) prediction algorithm given by M.Zuker. The algorithm predicts the possible secondary structures based on minimum free energy $(\Delta G)$ criterion. We arranged the secondary structures according to increasing free energies (a negative quantity), and selected the first 20 which had the least free energy values.

As we had hypothesized, our next experiment was to see whether the human Selenocysteine tRNA binds to the viral selenoprotein mRNA. For this the tertiary structures of the above 20 secondary structures were designed using computational tools. Human selenocysteine tRNA sequence was obtained from NCBI and secondary structure is designed. The tertiary structure of human Selenocysteine tRNA was obtained from PDB (PDB id 3A3A). We then tried to dock them individually, i.e. we performed twenty dockings, with the 20 tertiary structures of SECIS elements as receptor and human Selenocysteine tRNA as the ligand.

In the last part of our work, we removed the residues A8 and U9 from the tertiary structure of human Selenocysteine tRNA and performed the dockings again. The new docking results were compared with the earlier docking results.

\section{Results and Discussion}

The 3' untranslated region of all the sequences showed the presence of SECIS elements (Table 1). 
Table 1. Motifs found in 3' UTR of HIV-1 genome sequences.

\begin{tabular}{|c|c|c|c|}
\hline Strain & Secis Type-1 & Strain & Secis Type-1 \\
\hline $\begin{array}{c}\text { gi } 217038387|\mathrm{gb}| \mathrm{FJ} 460501.1 \mid \text { HIV-1 isolate HK004 from } \\
\text { Hong Kong, complete genome }\end{array}$ & $\checkmark$ & $\begin{array}{c}\text { gi| } 170878295|\mathrm{gb}| \text { EU } 541617.1 \mid \text { HIV-1 clone pIIIB from } \\
\text { USA, complete genome }\end{array}$ & $\checkmark$ \\
\hline $\begin{array}{l}\text { gi|13540181|gb|AF289550.1| HIV-1 clone 96TZ-BF110 } \\
\text { from Tanzania, complete genome }\end{array}$ & $\checkmark$ & $\begin{array}{l}\text { gi|161334695|gb|EU220698.1| HIV-1 isolate 04CA7750 } \\
\text { from Canada, complete genome }\end{array}$ & $\checkmark$ \\
\hline $\begin{array}{l}\text { gi|167651353|gb|EU293450.1| HIV-1 isolate 99ZALT46 } \\
\text { from South Africa, complete genome }\end{array}$ & $\checkmark$ & $\begin{array}{c}\text { gi|117940228|gb|DQ912823.1| HIV-1 isolate MA from } \\
\text { Denmark, complete genome }\end{array}$ & $\checkmark$ \\
\hline $\begin{array}{l}\text { gi|213495604|gb|FJ195091.1| HIV-1 isolate BREPM1081 } \\
\text { from Brazil, complete genome }\end{array}$ & $\checkmark$ & $\begin{array}{l}\text { gi|168208535|gb|EU448296.1| HIV-1 strain 06FR-CRN } \\
\text { from France, complete genome }\end{array}$ & $\checkmark$ \\
\hline $\begin{array}{l}\text { gi|212674726|gb|EU884501.1| HIV-1 isolate ES P1423 } \\
\text { (CRF02_AG) from Spain, complete genome }\end{array}$ & $\checkmark$ & $\begin{array}{l}\text { gi|164415926|gb|DQ020274.2| HIV-1 isolate CB134 from } \\
\text { Cuba, complete genome }\end{array}$ & $\checkmark$ \\
\hline $\begin{array}{l}\text { gi|195409392|gb|EU697909.1| HIV-1 isolate J11456 from } \\
\text { Saudi Arabia, complete genome }\end{array}$ & $\checkmark$ & $\begin{array}{l}\text { gi }|157885655| \mathrm{gb}|\mathrm{EU} 031915.1| \mathrm{HIV}-1 \text { isolate 07MYKLD49 } \\
\text { from Malaysia, complete genome }\end{array}$ & $\checkmark$ \\
\hline $\begin{array}{l}\text { gi }|83026775| \mathrm{gb}|\mathrm{DQ} 295192.1| \mathrm{HIV}-1 \text { isolate 04LSK7 } \\
\text { from South Korea, complete genome }\end{array}$ & $\checkmark$ & $\begin{array}{l}\text { gi|125541773|gb|EF192591.1| HIV-1 isolate CU-98-26 } \\
\text { from Thailand, complete genome }\end{array}$ & $\checkmark$ \\
\hline $\begin{array}{l}\text { gi|197257781 |gb|EU693240.1| HIV-1 isolate } \\
\text { 06CM-BA-040 from Cameroon, complete genome }\end{array}$ & $\checkmark$ & $\begin{array}{c}\text { gi }|85035359| \mathrm{gb}|\mathrm{DQ} 230841.1| \mathrm{HIV}-1 \text { isolate TW_D3 from } \\
\text { Taiwan, complete genome }\end{array}$ & $\checkmark$ \\
\hline $\begin{array}{l}\text { gi|194500414|gb|EU861977.1| HIV-1 isolate } 60000 \text { from } \\
\text { Italy, complete genome }\end{array}$ & $\checkmark$ & $\begin{array}{l}\text { gi|117643970|gb|EF029069.1| HIV-1 isolate } \\
\text { U.NL.01.H10986_C11 from Netherlands, complete genome }\end{array}$ & $\checkmark$ \\
\hline $\begin{array}{l}\text { gi }|209156839| \mathrm{gb}|\mathrm{FJ} 213780.1| \mathrm{HIV}-1 \text { isolate UY05_4752 } \\
\text { from Uruguay, complete genome }\end{array}$ & $\checkmark$ & $\begin{array}{c}\text { gi }|51980229| \mathrm{gb} \mid \text { AY } 612637.1 \mid \mathrm{HIV}-1 \text { isolate PT2695 from } \\
\text { Portugal, complete genome }\end{array}$ & $\checkmark$ \\
\hline $\begin{array}{l}\text { gi|2944126|gb|U71182.1|HIVU71182 HIV-1 isolate } \\
\text { RL42 from China, complete genome }\end{array}$ & $\checkmark$ & $\begin{array}{l}\text { gi }|112497950| \mathrm{gb}|\mathrm{DQ} 676887.1| \mathrm{HIV}-1 \text { isolate } \\
\text { PS4048_Day143 from Australia, complete genome }\end{array}$ & $\checkmark$ \\
\hline $\begin{array}{l}\text { gi|158967436|gb|EU110097.1| HIV-1 isolate } \\
\text { ML1990PCR from Kenya, complete genome }\end{array}$ & $\checkmark$ & $\begin{array}{c}\text { gi }|63081177| \mathrm{gb}|\mathrm{AY} 968312.1| \mathrm{HIV}-1 \text { isolate ARE195FL } \\
\text { from Argentina, complete genome }\end{array}$ & $\checkmark$ \\
\hline $\begin{array}{c}\text { gi|6651466|gb|AF193277.1| HIV-1 isolate RU98001 from } \\
\text { Russia, complete genome }\end{array}$ & $\checkmark$ & $\begin{array}{l}\text { gi } 18643009|\mathrm{gb}| \mathrm{AY} 074891.1 \mid \mathrm{HIV}-1 \text { isolate 00BWMO35.1 } \\
\text { from Botswana, complete genome }\end{array}$ & $\checkmark$ \\
\hline $\begin{array}{l}\text { gi|3947925|gb|AF049337.1| HIV-1 CRF04_cpx clone } \\
\text { 94CY032-3 from Cyprus, complete genome }\end{array}$ & $\checkmark$ & $\begin{array}{l}\text { gi }|74099684| \mathrm{gb}|\mathrm{DQ} 083238.1| \mathrm{HIV}-1 \text { isolate } 1579 \mathrm{~A} \text { from } \\
\text { India, complete genome }\end{array}$ & $\checkmark$ \\
\hline $\begin{array}{l}\text { gi|62361768|gb|AY882421.1| HIV-1 isolate 9196/01 } \\
\text { from Germany, complete genome }\end{array}$ & $\checkmark$ & $\begin{array}{l}\text { gi|29409304|gb|AY093604.1| HIV-1 isolate 95SN7808 } \\
\text { from Senegal, complete genome }\end{array}$ & $\checkmark$ \\
\hline $\begin{array}{l}\text { gi }|18699247| \mathrm{gb}|\mathrm{AF} 414006.1| \mathrm{HIV}-1 \text { isolate } 98 \mathrm{BY} 10443 \\
\text { from Belarus, complete genome }\end{array}$ & $\checkmark$ & $\begin{array}{c}\text { gi }|47118239| \mathrm{gb} \mid \text { AY 536235.1| HIV-1 isolate CH12 from } \\
\text { Chile, complete genome }\end{array}$ & $\checkmark$ \\
\hline $\begin{array}{l}\text { gi|18699185|gb|AF413987.1| HIV-1 isolate 98UA0116 } \\
\text { from Ukraine, complete genome }\end{array}$ & $\checkmark$ & $\begin{array}{c}\text { gi|47118229|gb|AY536236.1| HIV-1 isolate V62 from } \\
\text { Venezuela, complete genome }\end{array}$ & $\checkmark$ \\
\hline $\begin{array}{l}\text { gi }|6466838| \mathrm{gb}|\mathrm{AF} 184155.1| \mathrm{HIV}-1 \mathrm{G} 829 \text { from Ghana } \\
\text { complete genome }\end{array}$ & $\checkmark$ & $\begin{array}{l}\text { gi }|38679157| \mathrm{gb}|\mathrm{AY} 352657.1| \mathrm{HIV}-1 \text { isolate UG266 from } \\
\text { Uganda, complete genome }\end{array}$ & $\checkmark$ \\
\hline $\begin{array}{l}\text { gi }|56131599| \mathrm{gb}|\mathrm{AY} 805330.1| \mathrm{HIV}-1 \text { isolate HIV1084i } \\
\text { from Zambia, complete genome }\end{array}$ & $\checkmark$ & $\begin{array}{l}\text { gi } 38679140|\mathrm{gb}| \text { AY352655.1| HIV-1 isolate SE9010 from } \\
\text { Sweden, complete genome }\end{array}$ & $\checkmark$ \\
\hline $\begin{array}{l}\text { gi }|6690753| \mathrm{gb}|\mathrm{AF} 197341.1| \mathrm{HIV}-1 \text { isolate 90CF4071 } \\
\text { from Central African Republic, complete genome }\end{array}$ & $\checkmark$ & $\begin{array}{l}\text { gi| } 14530226|\mathrm{gb}| \mathrm{AF} 286236.1 \mid \mathrm{AF} 286236 \mathrm{HIV}-1 \text { isolate } \\
\text { 83CD003 from Republic of the Congo, complete genome }\end{array}$ & $\checkmark$ \\
\hline $\begin{array}{c}\text { gi|17352343|gb|AY046058.1| HIV-1 from Greece, } \\
\text { complete genome }\end{array}$ & $\checkmark$ & $\begin{array}{l}\text { gi|3779261|gb|AF064699.1|AF064699 HIV-1 isolate } \\
\text { BFP90 from Burkina Faso, complete genome }\end{array}$ & $\checkmark$ \\
\hline $\begin{array}{l}\text { gi|13569307|gb|AF286233.1|AF286233 HIV-1 strain } \\
\text { 98IS002 from Israel, complete genome }\end{array}$ & $\checkmark$ & $\begin{array}{l}\mathrm{gi}|5668910| \mathrm{gb}|\mathrm{AF} 076474.1| \mathrm{AF} 076474 \text { HIV-1 isolate VI354 } \\
\text { from Gabon, complete genome }\end{array}$ & $\checkmark$ \\
\hline $\begin{array}{l}\text { gi } 6090965|\mathrm{gb}| \text { AF } 075703.1 \mid \mathrm{AF} 075703 \mathrm{HIV}-1 \text { isolate } \\
\text { FIN9363 subtype F1 from Finland, complete genome }\end{array}$ & $\checkmark$ & - & - \\
\hline
\end{tabular}

This result thus confirmed putatively, to some extent, that SECIS elements may be present in the HIV genome. Other motifs were also obtained i.e. K-Box, GY-Box, Gamma interferon activated inhibitor of Ceruloplasmin mRNA translation (GAIT element), Brd-Box, Cytoplas- mic polyadenylation element, Alcohol dehydrogenase 3'UTR down regulation control element (ADH_DRE), Mos polyadenylation response element (Mos-PRE), Androgen receptor CU-rich element (AR_CURE) in the 3' UTR and those in the 5' UTR are Terminal Oligo- 
pyrimidine Tract (TOP), Internal Ribosome Entry Site (IRES), Upstream Open Reading Frame (uORF).Exonic regulatory motifs, transcriptional regulatory motifs, miRNA target sites and RNA structural elements were also found, (see Table 2).

The presence of Selenocysteine insertion sequence (SECIS) elements has been confirmed in eukaryotes (including humans). In eukaryotes, SECIS elements are required for the expression of selenoproteins. Functional selenoproteins, similar to mammalian selenoproteins, have been found in the HIV-1 genome. Based on these already proven theories it was thought that, the HIV genome may contain SECIS elements and this was confirmed by performing a search for SECIS elements on all the 847 complete genome sequences of the HIV-1 genome. The number of SECIS elements obtained putatively was variable for each sequence. The total number of SECIS elements obtained was 3215.

Since lower free energy value means a highly stable structure, so out of the 3215 structures 25 most stable predicted structures were selected (see Figure 1).

The sequence of Human selenocysteine tRNA was retrieved from PDB (>3A3A:A|PDBID|CHAIN|SEQUENCEGCCCGGAUGAUCCUCAGUGGUCUGGGGUGCAGGCUUCAAACCUGUAGCUGUCUAGCGACAGAGUGGUUCAAUUCCACCUUUCGGGCGCCA) and its corresponding secondary structure was designed by application of RNA covariance models, which are general,

Table 2. Motifs found in regions other than 3' and 5'UTR.

\begin{tabular}{|c|c|c|c|c|}
\hline Strain & $\begin{array}{c}\text { Exonic } \\
\text { Regulatory Motifs }\end{array}$ & $\begin{array}{l}\text { Transcriptional } \\
\text { Regulatory Motifs }\end{array}$ & $\begin{array}{c}\text { miRNA } \\
\text { Target Sites }\end{array}$ & $\begin{array}{l}\text { RNA Structural } \\
\text { Elements }\end{array}$ \\
\hline $\begin{array}{c}\text { gi }|217038387| \mathrm{gb}|\mathrm{FJ} 460501.1| \text { HIV-1 isolate HK004 from Hong } \\
\text { Kong, complete genome }\end{array}$ & 54 & 17 & 111 & \\
\hline $\begin{array}{c}\text { gi|13540181|gb|AF289550.1| HIV-1 clone 96TZ-BF110 from } \\
\text { Tanzania, complete genome }\end{array}$ & 53 & 18 & 89 & \\
\hline $\begin{array}{l}\text { gi|167651353|gb|EU293450.1| HIV-1 isolate 99ZALT46 from } \\
\text { South Africa, complete genome }\end{array}$ & 52 & 21 & 97 & \\
\hline $\begin{array}{c}\text { gi } 213495604|\mathrm{gb}| \mathrm{FJ} 195091.1 \mid \mathrm{HIV}-1 \text { isolate BREPM1081 from } \\
\text { Brazil, complete genome }\end{array}$ & 54 & 19 & 109 & 1 \\
\hline $\begin{array}{l}\text { gi|212674726|gb|EU884501.1| HIV-1 isolate ES P1423 } \\
\text { (CRF02_AG) from Spain, complete genome }\end{array}$ & 47 & 19 & 93 & \\
\hline $\begin{array}{c}\text { gi|195409392|gb|EU697909.1| HIV-1 isolate J11456 from Saudi } \\
\text { Arabia, complete genome }\end{array}$ & 42 & 17 & 104 & \\
\hline $\begin{array}{c}\text { gi }|83026775| \mathrm{gb}|\mathrm{DQ} 295192.1| \mathrm{HIV}-1 \text { isolate 04LSK7 from South } \\
\text { Korea, complete genome }\end{array}$ & 47 & 20 & 108 & \\
\hline $\begin{array}{l}\text { gi|197257781|gb|EU693240.1| HIV-1 isolate 06CM-BA-040 } \\
\text { from Cameroon, complete genome }\end{array}$ & 48 & 16 & 88 & \\
\hline $\begin{array}{c}\text { gi|194500414|gb|EU861977.1| HIV-1 isolate } 60000 \text { from Italy, } \\
\text { complete genome }\end{array}$ & 51 & 19 & 96 & \\
\hline $\begin{array}{c}\text { gi|209156839|gb|FJ213780.1| HIV-1 isolate UY05_4752 from } \\
\text { Uruguay, complete genome }\end{array}$ & 49 & 16 & 89 & \\
\hline $\begin{array}{c}\text { gi|2944126|gb|U71182.1|HIVU71182 HIV-1 isolate RL42 from } \\
\text { China, complete genome }\end{array}$ & 56 & 17 & 103 & \\
\hline $\begin{array}{c}\text { gi }|158967436| \mathrm{gb} \mid \text { EU110097.1| HIV-1 isolate ML1990PCR from } \\
\text { Kenya, complete genome }\end{array}$ & 53 & 18 & 75 & \\
\hline $\begin{array}{c}\text { gi }|6651466| \mathrm{gb}|\mathrm{AF} 193277.1| \text { HIV-1 isolate RU98001 from } \\
\text { Russia, complete genome }\end{array}$ & 57 & 16 & 96 & \\
\hline $\begin{array}{l}\text { gi|3947925|gb|AF049337.1| HIV-1 CRF04_cpx clone } \\
\text { 94CY032-3 from Cyprus, complete genome }\end{array}$ & 56 & 18 & 105 & \\
\hline $\begin{array}{c}\text { gi } 170878295|\mathrm{gb}| \mathrm{EU} 541617.1 \mid \text { HIV-1 clone pIIIB from USA, } \\
\text { complete genome }\end{array}$ & 61 & 21 & 100 & \\
\hline $\begin{array}{l}\text { gi }|161334695| \mathrm{gb} \mid \text { EU220698.1| HIV-1 isolate 04CA7750 from } \\
\text { Canada, complete genome }\end{array}$ & 51 & 16 & 94 & \\
\hline $\begin{array}{c}\text { gi }|117940228| g b|\mathrm{DQ} 912823.1| \mathrm{HIV}-1 \text { isolate MA from } \\
\text { Denmark, complete genome }\end{array}$ & 54 & 19 & 93 & \\
\hline $\begin{array}{c}\text { gi }|168208535| \mathrm{gb} \mid \text { EU448296.1| HIV-1 strain 06FR-CRN from } \\
\text { France, complete genome }\end{array}$ & 52 & 25 & 88 & \\
\hline
\end{tabular}




\begin{tabular}{|c|c|c|c|c|}
\hline $\begin{array}{l}\text { gi|164415926|gb|DQ020274.2| HIV-1 isolate CB134 from } \\
\text { Cuba, complete genome }\end{array}$ & 42 & 20 & 105 & \\
\hline $\begin{array}{l}\text { gi } 157885655|\mathrm{gb}| \text { EU031915.1| HIV-1 isolate 07MYKLD49 } \\
\text { from Malaysia, complete genome }\end{array}$ & 51 & 17 & 96 & \\
\hline $\begin{array}{c}\text { gi } 125541773|\mathrm{gb}| \mathrm{EF} 192591.1 \mid \text { HIV-1 isolate CU-98-26 from } \\
\text { Thailand, complete genome }\end{array}$ & 50 & 24 & 95 & \\
\hline $\begin{array}{l}\text { gi } 85035359|\mathrm{gb}| \mathrm{DQ} 230841.1 \mid \mathrm{HIV}-1 \text { isolate TW_D3 from } \\
\text { Taiwan, complete genome }\end{array}$ & 52 & 22 & 91 & \\
\hline $\begin{array}{l}\text { gi }|117643970| \mathrm{gb}|\mathrm{EF} 029069.1| \mathrm{HIV}-1 \text { isolate } \\
\text { U.NL.01.H10986_C11 from Netherlands, complete genome }\end{array}$ & 51 & 19 & 89 & \\
\hline $\begin{array}{l}\text { gi }|51980229| \mathrm{gb} \mid \text { AY } 612637.1 \mid \text { HIV-1 isolate PT2695 from } \\
\text { Portugal, complete genome }\end{array}$ & 39 & 19 & 107 & \\
\hline $\begin{array}{l}\text { gi|112497950|gb|DQ676887.1| HIV-1 isolate PS4048_Day143 } \\
\text { from Australia, complete genome }\end{array}$ & 51 & 19 & 78 & \\
\hline $\begin{array}{c}\text { gi }|63081177| \mathrm{gb} \mid \text { AY9 } 968312.1 \mid \text { HIV-1 isolate ARE195FL from } \\
\text { Argentina, complete genome }\end{array}$ & 57 & 24 & 102 & \\
\hline $\begin{array}{l}\text { gi|18643009|gb|AY074891.1| HIV-1 isolate 00BWMO35.1 } \\
\text { from Botswana, complete genome }\end{array}$ & 54 & 20 & 109 & \\
\hline $\begin{array}{l}\text { gi }|23986250| \mathrm{gb}|\mathrm{AY} 049711.1| \mathrm{HIV}-1 \text { isolate 01IN565.14 from } \\
\text { India, complete genome }\end{array}$ & 51 & 18 & 95 & \\
\hline $\begin{array}{l}\text { gi }|46243163| \mathrm{gb} \mid \text { AY } 535660.1 \mid \text { HIV-1 isolate EE0369 from } \\
\text { Estonia, complete genome }\end{array}$ & 54 & 18 & 102 & \\
\hline $\begin{array}{l}\text { gi|62361768|gb|AY882421.1| HIV-1 isolate 9196/01 from } \\
\text { Germany, complete genome }\end{array}$ & 47 & 19 & 85 & \\
\hline $\begin{array}{c}\text { gi }|18699247| \mathrm{gb}|\mathrm{AF} 414006.1| \mathrm{HIV}-1 \text { isolate } 98 \mathrm{BY} 10443 \text { from } \\
\text { Belarus, complete genome }\end{array}$ & 55 & 17 & 104 & \\
\hline $\begin{array}{c}\text { gi }|18699185| \mathrm{gb}|\mathrm{AF} 413987.1| \mathrm{HIV}-1 \text { isolate } 98 \mathrm{UA} 0116 \text { from } \\
\text { Ukraine, complete genome }\end{array}$ & 47 & 19 & 99 & 1 \\
\hline $\begin{array}{l}\text { gi } 6466838|\mathrm{gb}| \mathrm{AF} 184155.1 \mid \mathrm{HIV}-1 \mathrm{G} 829 \text { from Ghana } \\
\text { complete genome }\end{array}$ & 47 & 20 & 87 & \\
\hline $\begin{array}{c}\text { gi }|56131599| \mathrm{gb} \mid \text { AY } 805330.1 \mid \text { HIV-1 isolate HIV1084i from } \\
\text { Zambia, complete genome }\end{array}$ & 50 & 21 & 100 & \\
\hline $\begin{array}{c}\text { gi }|29409304| \mathrm{gb} \mid \text { AY093604.1| HIV-1 isolate 95SN7808 from } \\
\text { Senegal, complete genome }\end{array}$ & 53 & 26 & 90 & \\
\hline $\begin{array}{c}\text { gi }|47118239| \mathrm{gb}|\mathrm{AY} 536235.1| \mathrm{HIV}-1 \text { isolate } \mathrm{CH} 12 \text { from Chile, } \\
\text { complete genome }\end{array}$ & 47 & 18 & 94 & 1 \\
\hline $\begin{array}{c}\text { gi }|47118229| \mathrm{gb} \mid \text { AY5536236.1| HIV-1 isolate V62 from } \\
\text { Venezuela, complete genome }\end{array}$ & 50 & 17 & 103 & \\
\hline $\begin{array}{l}\text { gi|38679157|gb|AY352657.1| HIV-1 isolate UG266 from } \\
\text { Uganda, complete genome }\end{array}$ & 57 & 21 & 100 & \\
\hline $\begin{array}{l}\text { gi|38679131|gb|AY352654.1| HIV-1 isolate SE8646 from } \\
\text { Sweden, complete genome }\end{array}$ & 43 & 19 & 101 & \\
\hline $\begin{array}{l}\text { gi|6690753|gb|AF197341.1| HIV-1 isolate 90CF4071 from } \\
\text { Central African Republic, complete genome }\end{array}$ & 50 & 22 & 93 & \\
\hline $\begin{array}{c}\text { gi }|17352343| \mathrm{gb}|\mathrm{AY} 046058.1| \mathrm{HIV}-1 \text { from Greece, complete } \\
\text { genome }\end{array}$ & 55 & 22 & 84 & \\
\hline $\begin{array}{l}\text { gi|14530226|gb|AF286236.1|AF286236 HIV-1 isolate } \\
\text { 83CD003 from Republic of the Congo, complete genome }\end{array}$ & 54 & 18 & 107 & \\
\hline $\begin{array}{l}\text { gi|3779261 |gb|AF064699.1|AF064699 HIV-1 isolate BFP90 } \\
\text { from Burkina Faso, complete genome }\end{array}$ & 50 & 12 & 91 & \\
\hline $\begin{array}{c}\text { gi|13569307|gb|AF286233.1|AF286233 HIV-1 strain 98IS002 } \\
\text { from Israel, complete genome }\end{array}$ & 53 & 24 & 85 & \\
\hline $\begin{array}{c}\text { gi|6090965|gb|AF075703.1|AF075703 HIV-1 isolate FIN9363 } \\
\text { subtype F1 from Finland, complete genome }\end{array}$ & 45 & 18 & 99 & \\
\hline $\begin{array}{l}\text { gi }|5668910| \mathrm{gb}|\mathrm{AF} 076474.1| \mathrm{AF} 076474 \mathrm{HIV}-1 \text { isolate VI354 } \\
\text { from Gabon, complete genome }\end{array}$ & 50 & 16 & 94 & \\
\hline
\end{tabular}




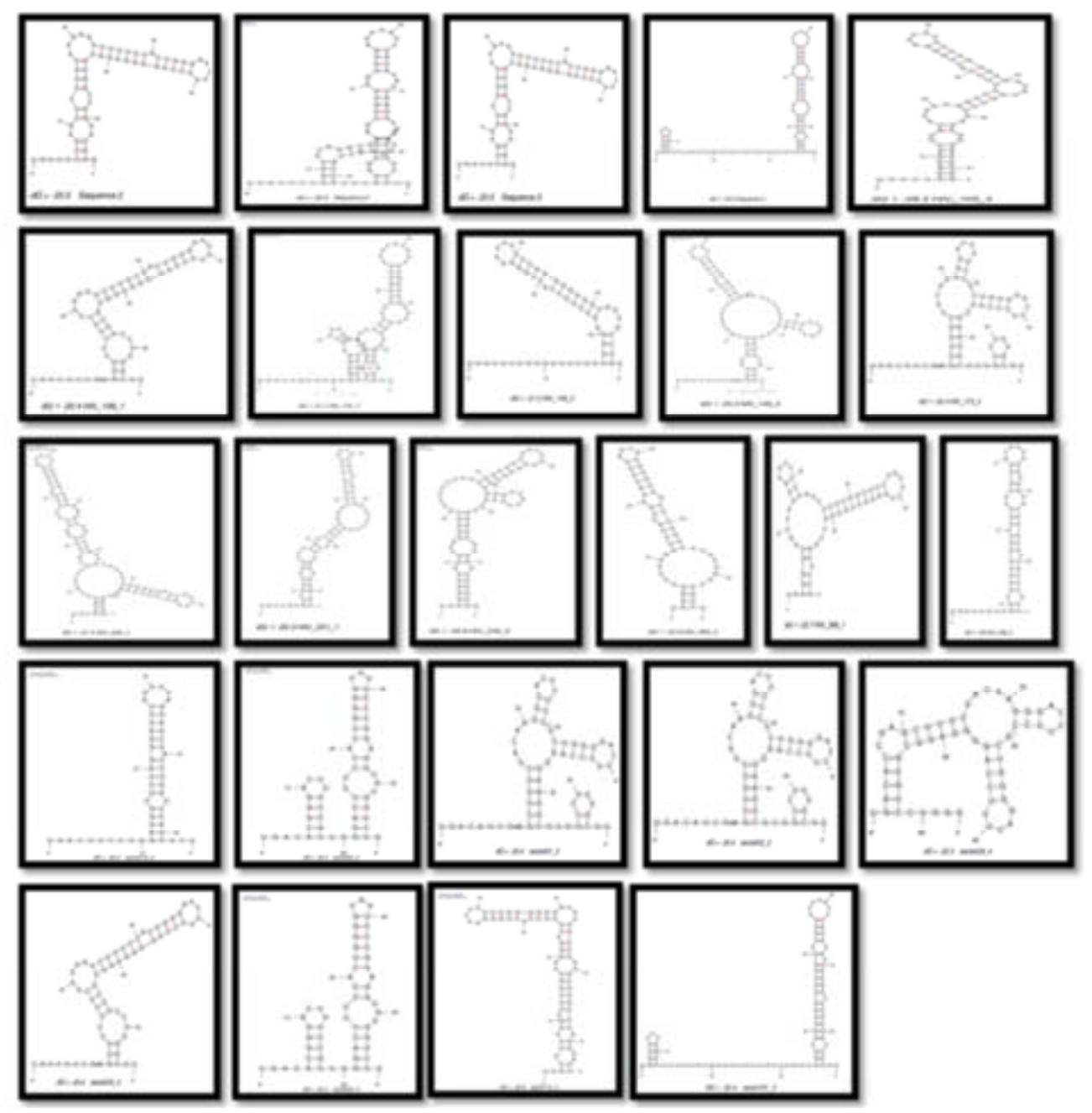

Figure 1. Predicted secondary structures of SECIS elements.

probabilistic secondary structure profiles based on stochastic context-free grammars (see Figure 2).

The tertiary structures of all the 20 SECIS elements showed a similar kind of a structure, (see Figure 3).

These were docked to the crystal structure of human Selenocysteine tRNA (see Figure 4).

Also known is the fact that during HIV infection selenium pool of the host gets depleted and viral selenoproteins increase. During translation, the tRNA binds to the mRNA (at the corresponding codon) for the expression of the protein. Human Selenocysteine tRNA $\left(\mathrm{tRNA}^{\mathrm{Sec}}\right)$ has an anticodon complementary to the UGA codon. If the human Selenocysteine tRNA binds to the viral mRNA that has the SECIS elements, then the viral selenoproteins might get expressed and this may be the probable cause of the increase in viral selenoproteins and depletion of host selenium, as it is being used up by the viral genome. So, the human Selenocysteine tRNA, instead of getting attached to its own selenoprotein mRNA,

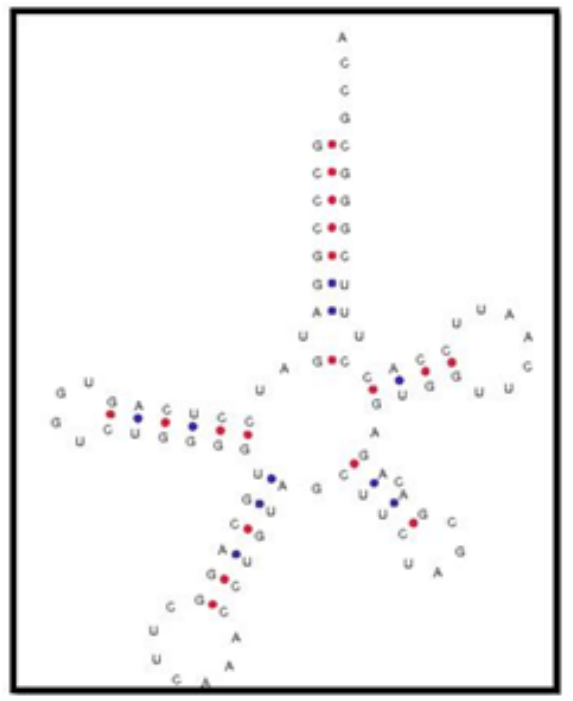

Figure 2. Predicted secondary structure of human selenocysteine tRNA. 

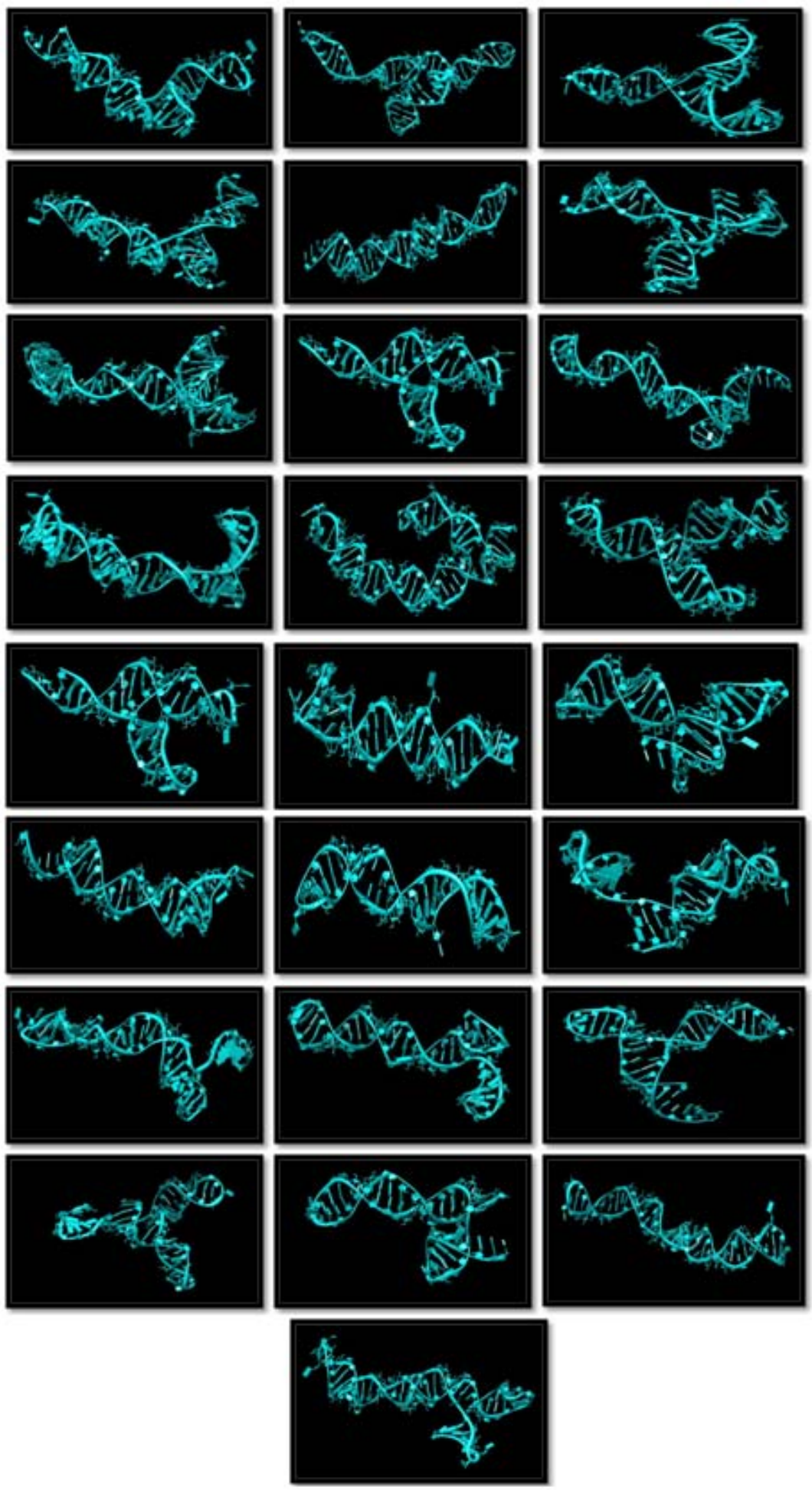

Figure 3. Predicted tertiary structures of SECIS elements.

attaches to the viral selenoprotein mRNA during HIV infection. The docking results confirmed this putatively as the free energy values of the 20 docked complexes (see Figure 5) were very low, hence the binding was highly stable.

The docked complexes were clustered according to E-values and country (Table 3).

The D stem and the extra arm do not form tertiary in- 
Table 3. Clustering according to E-values and country.

\begin{tabular}{|c|c|}
\hline Sequence Details & E Total \\
\hline >gi|217038387|gb|FJ460501.1| HIV-1 isolate HK004 from Hong Kong, complete genome & -26049.78 \\
\hline >gi|217038377|gb|FJ460500.1| HIV-1 isolate HK003 from Hong Kong, complete genome & -24043.97 \\
\hline >gi|217038367|gb|FJ460499.1| HIV-1 isolate HK002 from Hong Kong, complete genome & -27350.14 \\
\hline >gi|167651343|gb|EU293449.1| HIV-1 isolate 99ZALT45 from South Africa, complete genome & -37608.79 \\
\hline >gi|149939408|gb|EF633445.1| HIV-1 isolate R1 from South Africa, complete genome & -29866.54 \\
\hline >gi|63098379|gb|DQ011175.1| HIV-1 isolate 03ZASK005B2 from South Africa, complete genome & -19193.88 \\
\hline >gi|63098294|gb|DQ011166.1| HIV-1 isolate 04ZASK135B1 from South Africa, complete genome & -20754.28 \\
\hline$>\mathrm{gi}|85700643| \mathrm{gb}|\mathrm{DQ} 351234.1| \mathrm{HIV}-1$ isolate 03ZASK233B1 from South Africa, complete genome & -15853.64 \\
\hline$>$ gi|85700503|gb|DQ351220.1| HIV-1 isolate 02ZAPS006MB1 from South Africa, complete genome & -29420.57 \\
\hline >gi|68522063|gb|DQ093598.1| HIV-1 isolate 04ZAPS202B1 from South Africa, complete genome & -16265.70 \\
\hline >gi|51572093|gb|AY703908.1| HIV-1 isolate 03ZASK040B1 from South Africa, complete genome & -15903.14 \\
\hline >gi|46486663|gb|AY585268.1| HIV-1 isolate C.ZA.1069MB from South Africa, complete genome & -29545.00 \\
\hline >gi|57338555|gb|AY838567.1| HIV-1 isolate 1069MB from South Africa, complete genome & -16336.54 \\
\hline$>\mathrm{gi}|24181477| \mathrm{gb}|\mathrm{AF} 411964.1| \mathrm{HIV}-1$ isolate 99ZACM4 from South Africa, complete genome & -34332.66 \\
\hline >gi|29119285|gb|AY173954.1| HIV-1 isolate US3 from USA, complete genome & -22281.61 \\
\hline >gi|37677763|gb|AY331283.1| HIV-1 isolate 1001-09 from USA, complete genome & -30852.84 \\
\hline >gi|37677753|gb|AY331282.1| HIV-1 isolate 1001-07 from USA, complete genome & -18468.71 \\
\hline >gi|55735993|gb|AY771593.1| HIV-1 isolate BREPM278 from Brazil, complete genome & -27615.47 \\
\hline >gi|55735957|gb|AY771589.1| HIV-1 isolate BREPM108 from Brazil, complete genome & -17908.92 \\
\hline >gi|157274079|gb|EF637057.1| HIV-1 isolate BREPM1023 from Brazil, complete genome & -38704.25 \\
\hline >gi|157274021|gb|EF637051.1| HIV-1 isolate BREPM1032 from Brazil, complete genome & -16073.70 \\
\hline >gi|157274001|gb|EF637049.1| HIV-1 isolate BREPM1035 from Brazil, complete genome & -21868.21 \\
\hline >gi|86277616|gb|DQ358809.1| HIV-1 isolate 02BR011 from Brazil, complete genome & -44666.35 \\
\hline >gi|221474|dbj|D10112.1|HIVCAM1 Human immunodeficiency virus 1 proviral DNA, complete genome & -32795.28 \\
\hline
\end{tabular}

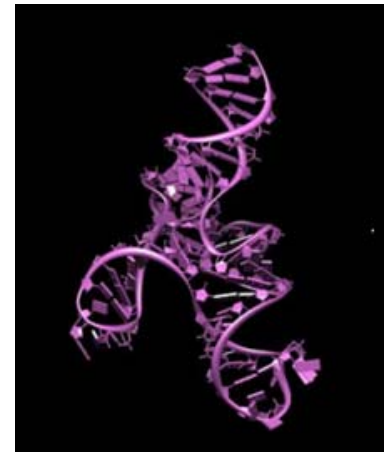

Figure 4. Predicted crystal structure of human selenocysteine tRNA. teractions in $\mathrm{tRNA}^{\mathrm{Sec}}$. Rather, $\mathrm{tRNA}^{\mathrm{Sec}}$ has an open cavity, in place of the tertiary core of a canonical tRNA. The linker residues, A8 and U9, connecting the acceptor and D stems, are not involved in tertiary base pairing. Instead, U9 is stacked on the first base pair of the extra arm. These features might allow tRNA ${ }^{\text {Sec }}$ to be the target of the Selenocysteine synthesis/incorporation machineries. Following this finding, the residues A8 and U9 were removed from the structure of human selenocysteine tRNA and this was docked with the SECIS element (one from Group-3).The atoms of the residues have been highlighted (see Figure 6) and the bond between the SECIS element and the residues is shown (see Figure 7). 

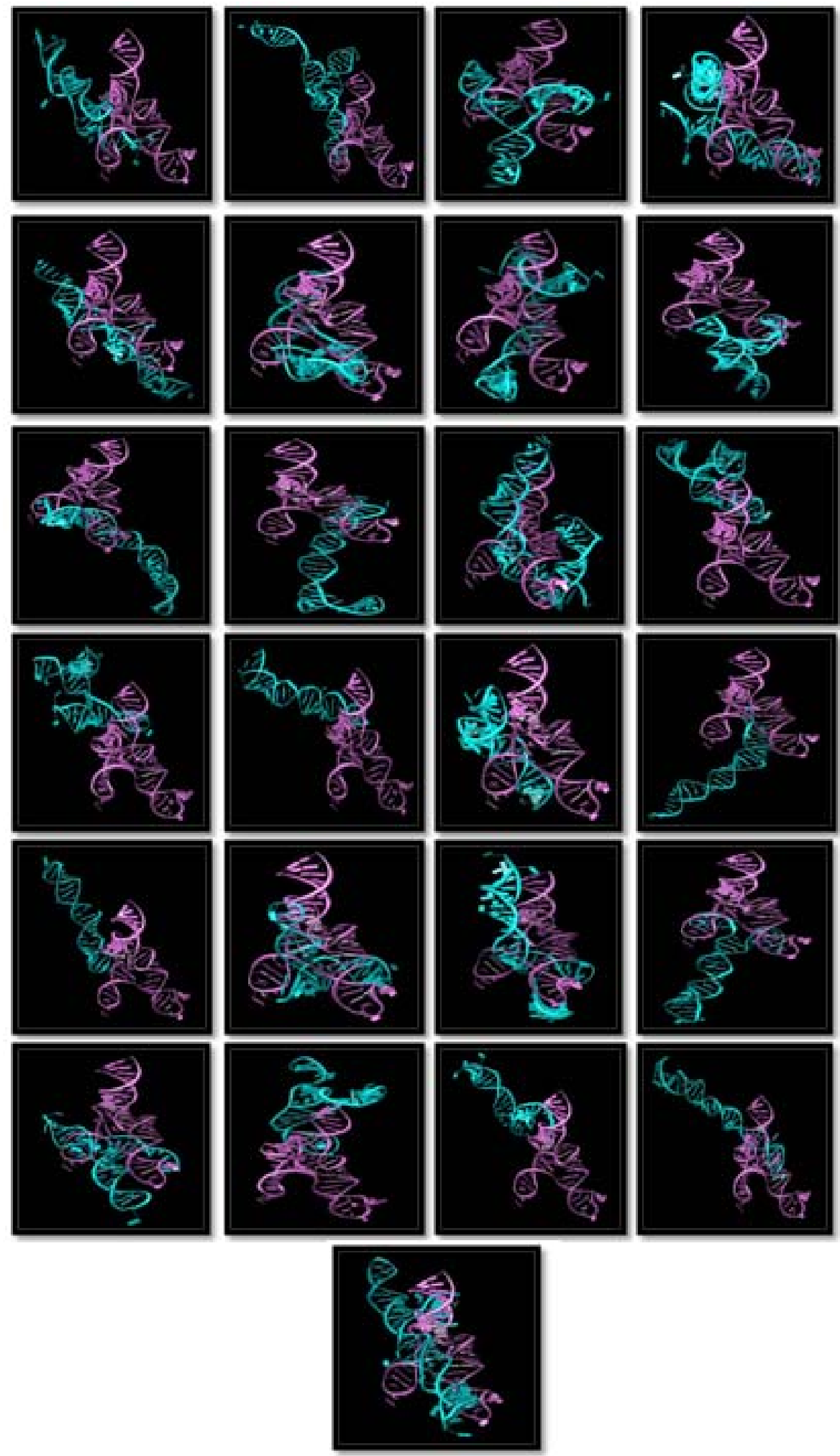

Figure 5. Predicted 20 docked complexes.

The result showed an increase in the E-value i.e. the free energy, hence a less stable structure than was ob- tained earlier (see Figure 8).

It shows that the residues A8 and U9 in the open cav- 


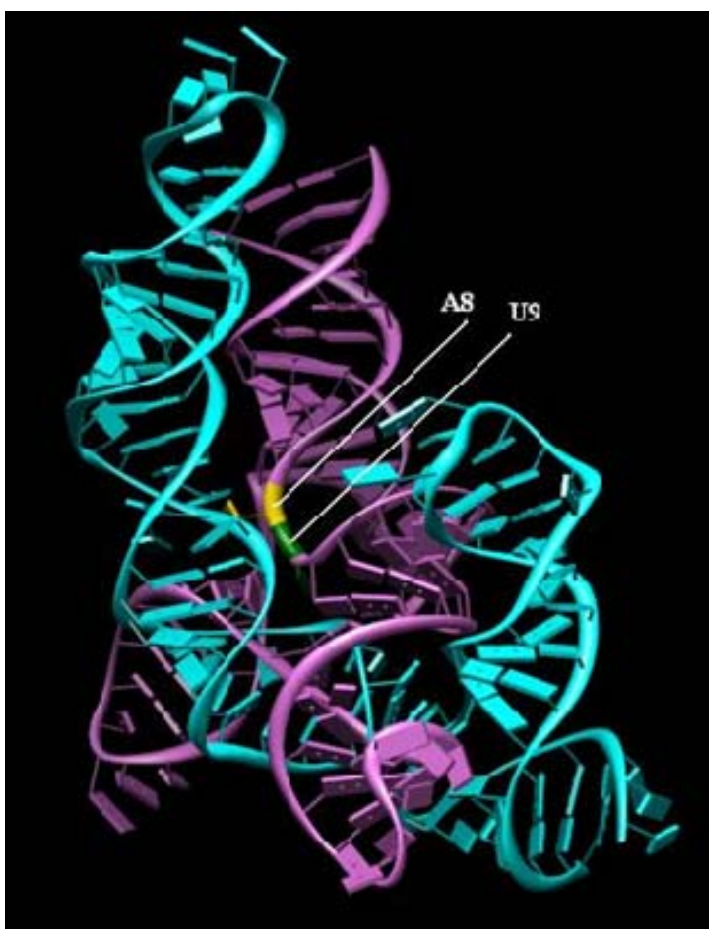

Figure 6. Residues A8 and U9 of the predicted structures have been highlighted.

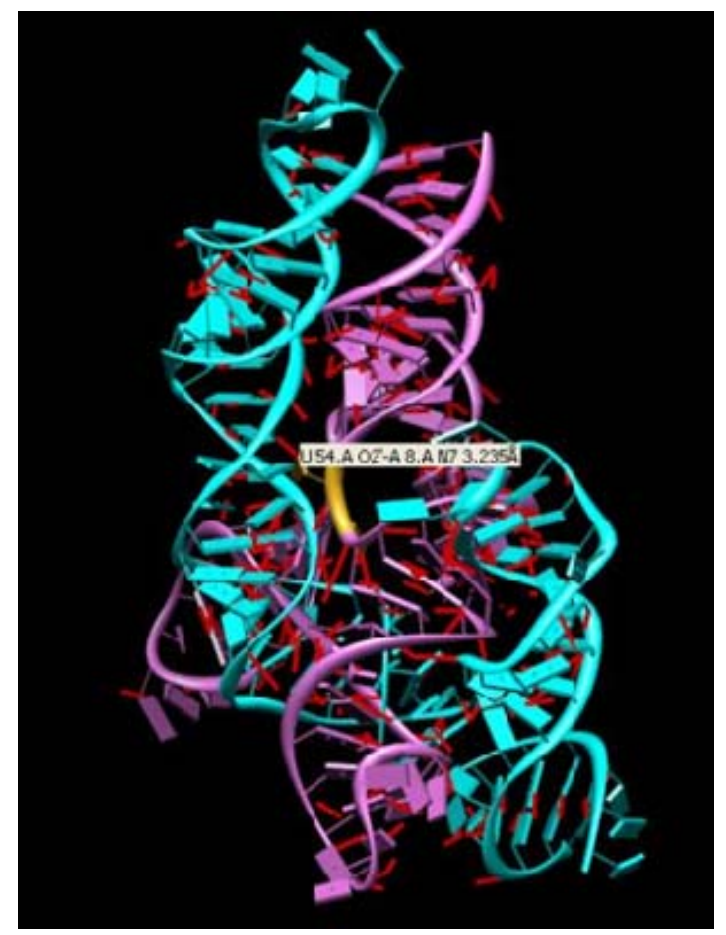

Figure 7. Bond between the SECIS element and the residues A8 and U9 of the predicted structures is shown.

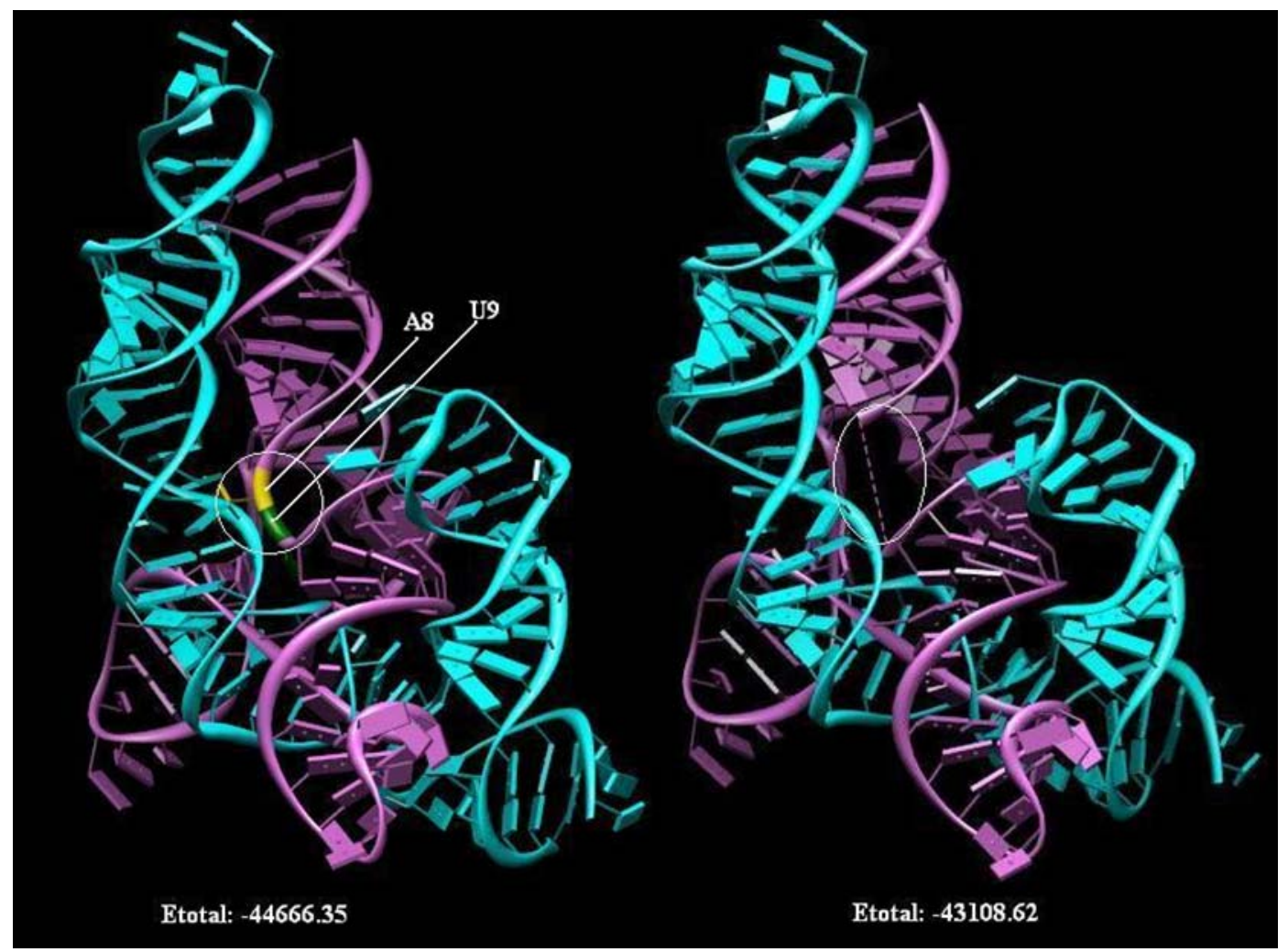

Figure 8. Showing docked complexes with and without the residues A8 and U9 of the predicted structures and the respective E-values. 
ity are an important part of the stable binding of the human Sec tRNA ${ }^{\mathrm{Sec}}$ and HIV SECIS elements.

\section{REFERENCES}

[1] V. N. Gladyshev, T. C. Stadtman, D. L. Hatfield and K. T. Jeang, "Levels of Major Selenoproteins in T Cells Decrease during HIV Infection and Low Molecular Mass Selenium Compounds Increase," Proceedings of the National Academy of Sciences of the United States of America, Vol. 96, No. 3, 1999, pp. 835-839.

doi:10.1073/pnas.96.3.835

[2] B. Moghadaszadeh and A. H. Beggs, "Selenoproteins and Their Impact on Human Health through Diverse Physiological Pathways," Physiology, Vol. 21, No. 5, 2006, pp. 307-315. doi:10.1152/physiol.00021.2006

[3] B. M. Dworkin, "Selenium Deficiency in HIV Infection and the Acquired Immunodeficiency Syndrome (AIDS)," Chemico-Biological Interactions, Vol. 9, No. 2-3, 1994, pp. 181-186. doi:10.1016/0009-2797(94)90038-8

[4] J. Lu and A. Holmgren, "Selenoproteins," Journal of Biological Chemistry, Vol. 284, No. 2, 2009, pp.723-727. doi:10.1074/jbc.R800045200

[5] S. C. Gamble, A. Wiseman and P. S. Goldfarb, "Selenium-Dependent Glutathione Peroxidase and Other Selenoproteins: Their Synthesis and Biochemical Roles," Journal of Chemical Technology and Biotechnology, Vol. 68, No.2, 1997, pp. 123-134. doi:10.1002/(SICI)1097-4660(199702)68:2<123::AID-JC TB641>3.0.CO;2-O

[6] X. M. Xu, B. A. Carlson, Y. Zhang, H. Mix, G. V. Kryukov, R. S. Glass, M. J. Berry, V. N. Gladyshev and D. L. Hatfield, "New Developments in Selenium Biochemistry: Selenocysteine Biosynthesis in Eukaryotes and Archaea," Biological Trace Element Research, Vol. 119, No. 3, 2007, pp. 234-241. doi:10.1007/s12011-007-8003-9

[7] J. M. Coffin, S. H. Hughes and H. E. Varmus, "Retroviruses," Cold Spring Harbor Laboratory Press, New York, 1997.

[8] A. D. Frankel and J. A. Young, "HIV-1: Fifteen Proteins and an RNA," Annual Review of Biochemistry, Vol. 67, No. 1, 1998, pp. 1-25. doi:10.1146/annurev.biochem.67.1.1

[9] C. K. Damgaard, E. S. Andersen, B. Knudsen, J. Gorod- kin and J. Kjems, "RNA Interactions in the 5' Region of the HIV-1 Genome," Journal of Molecular Biology, Vol. 336, No. 2, 2004, pp. 369-379. doi:10.1016/j.jmb.2003.12.010

[10] S. P. Goff, "Host Factors Exploited by Retroviruses," Nature Reviews Microbiology, Vol. 5, No. 4, 2007, pp. 253-263.

[11] K. A. Wilkinson, R. J. Gorelick, S. M. Vasa, N. Guex, A. Rein, D. H. Mathews, M. C. Giddings and K. M. Weeks, "High-Throughput SHAPE Analysis Reveals Structures in HIV-1 Genomic RNA Strongly Conserved across Distinct Biological States," PLoS Biology, Vol. 6, No. 4, 2008, pp. 883-899. doi:10.1371/journal.pbio.0060096

[12] J. M. Watts, K. K. Dang, R. J. Gorelick, C. W. Leonard, J. W. Bess, R. Swanstrom Jr., C. L. Burch and K. M. Weeks, "Architecture and Secondary Structure of an Entire HIV-1 RNA Genome," Nature, Vol. 460, No. 7256, 2009, pp. 711-716. doi:10.1038/nature08237

[13] L. Zhao, A. G. Cox, J. A. Ruzicka, A. A. Bhat, W. Zhang and E. W. Taylor, "Molecular Modeling and in Vitro Activity of an HIV-1-Encoded Glutathione Peroxidase," Proceedings of the National Academy of Sciences of the United States of America, Vol. 97, No. 12, 2000, pp. 6356-6361. doi:10.1073/pnas.97.12.6356

[14] G. Leslie, "Potential SECIS Elements in HIV-1 Strain HXB2," Journal of Acquired Immune Deficiency Syndromes and Human Retrovirology, Vol. 17, No. 5, 1998, pp. 398-403. doi:10.1097/00042560-199804150-00003

[15] Y. Zhang and V. N. Gladyshev, "An Algorithm for Identification of Bacterial Selenocysteine Insertion Sequence Elements and Selenoprotein Genes," Bioinformatics, Vol. 21, No. 11, 2005, pp. 2580-2589. doi:10.1093/bioinformatics/bti400

[16] M. Zuker, "Mfold Web Server for Nucleic Acid Folding and Hybridization Prediction," Nucleic Acids Research, Vol. 31, No. 13, 2003, pp. 3406-3415. doi:10.1093/nar/gkg595

[17] Y. Ding and C. E. Lawrence, "A Bayesian Statistical Algorithm for RNA Secondary Structure Prediction," Computers \& Chemistry, Vol. 23, No. 3-4, 1999, pp. $387-$ 400. doi:10.1016/S0097-8485(99)00010-8

[18] Y. Itoh, S. Chiba, S. Sekine and S. Yokoyama, "Crystal Structure of Human Selenocysteine tRNA," Nucleic Acids Research, Vol. 37, No. 18, 2009, pp. 6259-6268. doi:10.1093/nar/gkp648 